\title{
Adipofascial Anterolateral Thigh Flap Safety: Applications and Complications
}

\author{
Tommaso Agostini ${ }^{1}$, Giulia Lo Russo ${ }^{2}$, Yi Xin Zhang ${ }^{3}$, Giuseppe Spinelli ${ }^{1}$, Davide Lazzeri ${ }^{4}$ \\ ${ }^{1}$ Maxillofacial Surgery Unit, Hospital of Florence, Florence; ${ }^{2}$ Plastic Surgery Unit, University Hospital of Florence, Florence, Italy; \\ ${ }^{3}$ Department of Plastic and Reconstructive Surgery, Shanghai Ninth People's Hospital, Shanghai Jiao Tong University School of Medicine, \\ Shanghai, China; ${ }^{4}$ Plastic and Reconstructive Surgery Unit, Hospital of Pisa, Pisa, Italy
}

Background A thinned anterolateral thigh (ALT) flap is often harvested to achieve optimal skin resurfacing. Several techniques have been described to thin an ALT flap including an adipocutaneous flap, an adipofascial flap and delayed debulking.

Methods By systematically reviewing all of the available literature in English and French, the present manuscript attempts to identify the common surgical indications, complications and donor site morbidity of the adipofascial variant of the ALT flap. The studies were identified by performing a systematic search on Medline, Ovid, EMBASE, the Cochrane Database of Systematic Reviews, Current Contents, PubMed, Google, and Google Scholar.

Results The study selection process was adapted from the Preferred Reporting Items for Systematic Reviews and Meta-Analyses statement, and 15 articles were identified using the study inclusion criteria. These articles were then reviewed for author name(s), year of publication, flap dimensions and thickness following defatting, perforator type, type of transfer, complications, thinning technique, number of cases with a particular area of application and donor site morbidity.

Conclusions The adipofascial variant of the ALT flap provides tissue to fill large defects and improve pliability. Its strong and safe blood supply permits adequate immediate or delayed debulking without vascular complications. The presence of the deep fascia makes it possible to prevent sagging by suspending and fixing the flap for functional reconstructive purposes (e.g., the intraoral cavity). Donor site morbidity is minimal, and thigh deformities can be reduced through immediate direct closure or liposuction and direct closure. A safe blood supply was confirmed by the rate of secondary flap debulking.

Keywords Free flaps / Microsurgery / Systematic review
Correspondence: Yi Xin Zhang Department of Plastic and Reconstructive Surgery, Shanghai Ninth People's Hospital, Shanghai Jiao Tong University School of Medicine, 639 Zhi Zao Ju Road, Shanghai 200011, China

Tel: +86-21-23271699

Fax: +86-21-63051858

E-mail: zhangyixin6688@hotmail.com
No potential conflict of interest relevant to this article was reported.

\section{INTRODUCTION}

Song et al. [1] described the vascular anatomy of the anterolateral, anteromedial and posterior thigh flaps in 1984. They harvested the anterolateral thigh (ALT) flap in 9 patients suffering from head and neck contractures following burn injuries. However, the ALT flap is now known to require a thickness adjustment to fit the defects being corrected, particularly in Caucasians $[1,2]$. To achieve good aesthetic and functional reconstructive outcomes, the proper thickness of the anatomical area to be re-

Copyright $\odot 2013$ The Korean Society of Plastic and Reconstructive Surgeons

This is an Open Access article distributed under the terms of the Creative Commons Attribution Non-Commercial License (http://creativecommons.org/

licenses/by-nc/3.0/) which permits unrestricted non-commercial use, distribution, and reproduction in any medium, provided the original work is properly cited.

www.e-aps.org 
constructed is used to determine the appropriate flap thickness. With this aim, several techniques have been described to thin the ALT flap, including the adipocutaneous flap, the adipofascial flap and delayed debulking. Herein, we review the ALT flap adipofascial configuration with special attention to the specific complications. By systematically reviewing all of the available papers, the present manuscript attempts to identify the common surgical indications, complications, and donor site morbidity of the adipofascial variant of the ALT flap.

\section{METHODS}

\section{Study design}

In this systematic literature review, we evaluated all of the available papers focusing on the adipofascial ALT flap and collected the following data: author name(s), year of publication, flap dimensions and thickness following defatting, perforator type, type of transfer, complications, thinning technique, number of cases with a particular area of application and donor site morbidity. All of these data were considered essential to properly investigate the adipofascial ALT flap. The published studies were searched and reviewed, and the data were collected as follows.

\section{Search strategy}

The studies were identified by performing a systematic search of Medline, Ovid, EMBASE, the Cochrane Database, Current Contents, PubMed, Google, and Google Scholar. The English and French versions of the identified articles and conference abstracts were then cross-referenced. The search terms used for the online search included anterolateral thigh flap, thinned anterolateral thigh flap, ultra-thinned anterolateral thigh flap, super-thinned anterolateral thigh flap, thinned flap, ultra-thinned flap, superthinned flap, thin flap, micro-dissected thinned flap and thinned flap, adipofascial flap and adipofascial anterolateral thigh flap. The results were then screened for titles and abstracts. When in doubt about the inclusion in our work, we reviewed the entire article for the adopted inclusion criteria (Table 1). The references were checked to identify additional papers. A flowchart of the systematic article selection is shown in Fig. 1. The data collection was updated on April 30, 2012.

\section{RESULTS}

The literature search yielded a total of 690 articles that were published between April 1984 and April 2012. We identified 15 articles fitting the inclusion criteria. Data collection was performed according to the inclusion and exclusion criteria (Table 1), and the results are summarised in Table 2. Fifteen papers were identified that reported on the immediate thinning of the adipofascial ALT flap technique and that were published between 2000 and 2011 [3-17].

Fig. 1. Flowchart of the systematic article selection performed

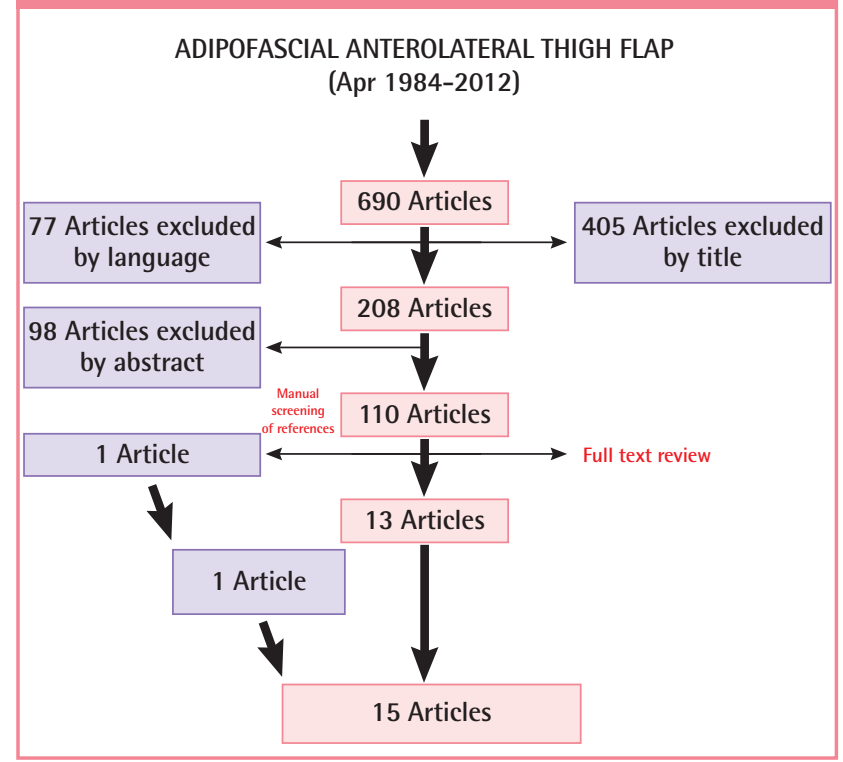

Table 1. Inclusion and exclusion criteria of the systematic literature review

\begin{tabular}{|ll|}
\hline Inclusion criteria & Exclusion criteria \\
\hline Human & Cadaver and anatomical study \\
English and French language papers & Experimental \\
At least the description of a case of a thinned anterolateral thigh flap & Split-flaps and through-and-through defects reconstructions \\
Review papers or case series or case report & Composite flaps (fasciocutaneous/musculocutaneous flaps, chimeric flaps) \\
Large/small anterolateral thigh flaps series, cases report, or letters to the editor & Harvesting techniques \\
Adipofascial anterolateral thigh flap & Donor site complications \\
Head and neck, trunk, or limb reconstructions & Donor site morbidity assessment \\
Pedicle and free flaps & Free flap monitoring techniques \\
& Microvascular anastomosis harvesting \\
& Adipocutaneous anterolateral thigh flap \\
& Perforator investigation methods (Doppler, computed tomography, magnetic \\
\end{tabular}




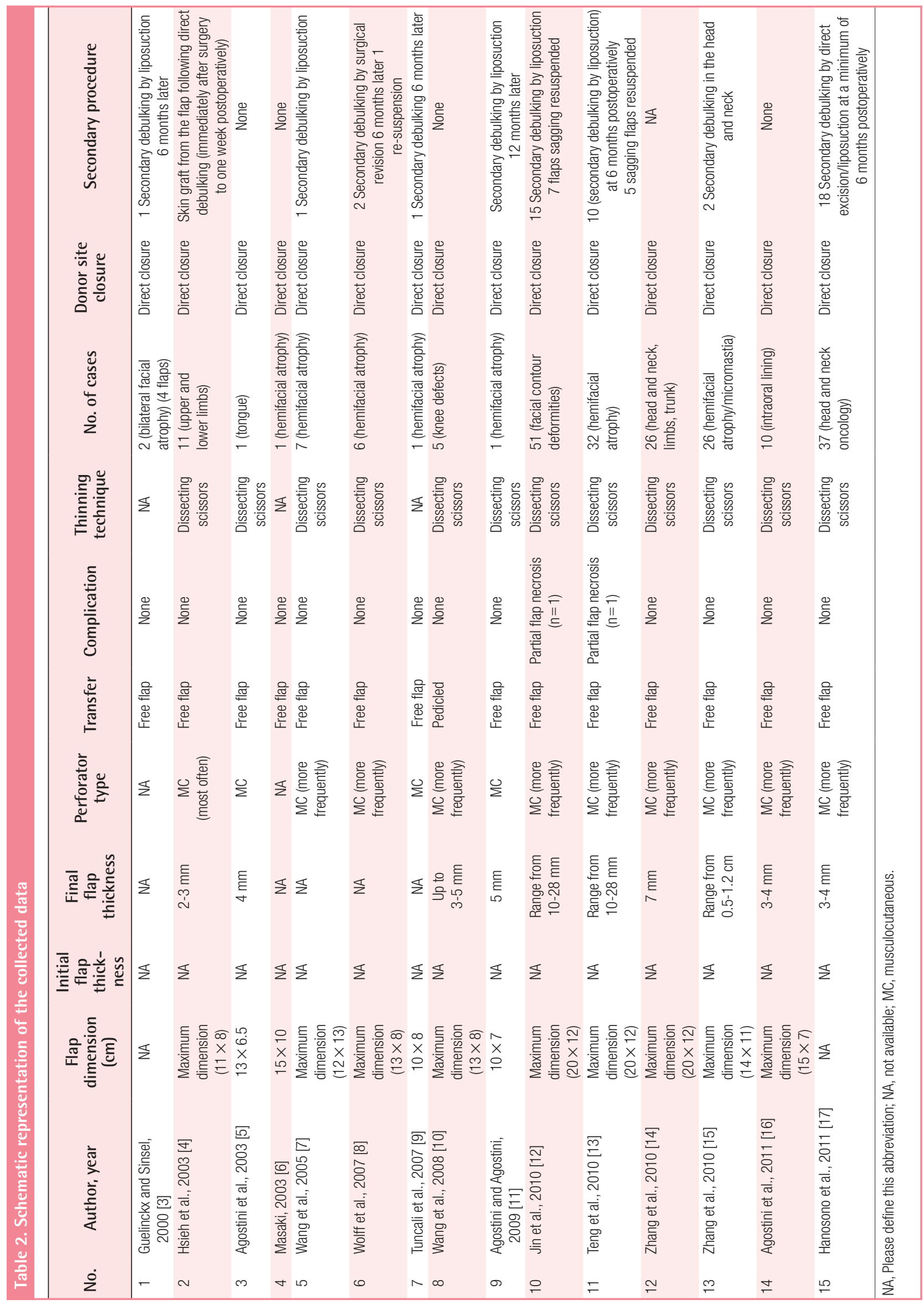


Fig. 2. Breakdown of the specific complications of the anterolateral thigh flap

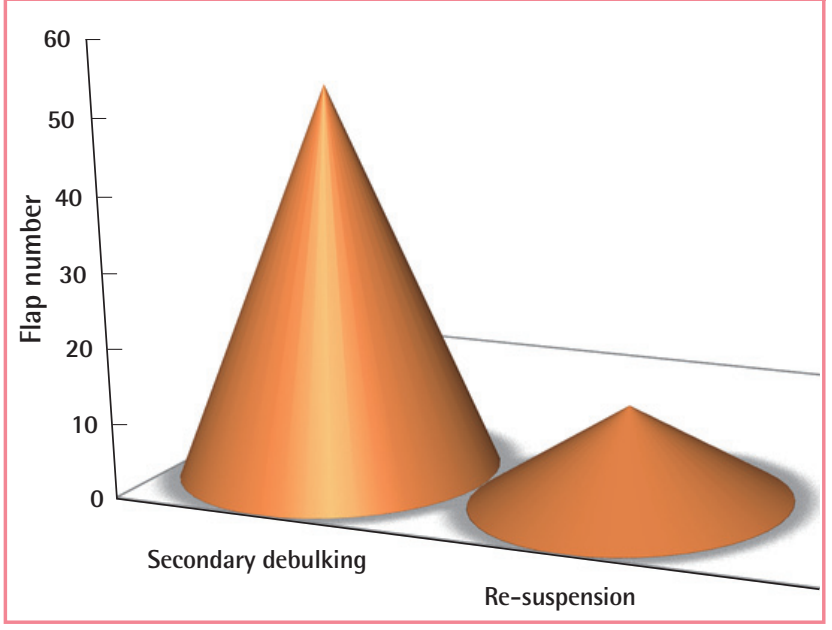

The flap size dimension was reported in 13 papers [4-16] and ranged from 70 to $240 \mathrm{~cm}^{2}$ (mean flap area, $140 \mathrm{~cm}^{2}$ ); it was not available in two cases $[3,17]$. The initial flap thickness was unavailable in all of the papers [3-17]. The reported flap thickness following defatting ranged from 0.5 to $2.8 \mathrm{~cm}$ (mean flap thickness, $0.64 \mathrm{~cm}$ ) [4,5,10-17] and was not available in 5 papers [3,6-9]. Musculocutaneous perforators were used in all the cases during flap rising, but the type of perforator was not available in 2 papers $[3,6]$. ALT flaps were transferred as free flaps in 14 papers [4-9,11-17], with the exception of Wang and co-workers, who harvested pedicled ALT flaps for knee defects [10]. All authors performed defatting using dissecting scissors before pedicle ligation to achieve proper haemostasis [4-8,10$17]$, but the defatting technique was not available in 3 cases $[3,6,9]$. Thinning-related complications were reported in two papers and consisted of 2 cases of partial liponecrosis that did not require further revision $[12,13]$. A total of 175 adipofascial ALT flaps were harvested for head and neck reconstruction [3,5-17]: 127 flaps were used for monolateral [6-9,12,13,15] or bilateral [3] facial atrophy correction, 37 flaps were used to repair post-oncologic defects of the head and neck [17], and 11 flaps were harvested to repair intraoral defects $[5,16]$. Eleven flaps were harvested for lower limb reconstruction as free [4] or pedicled [10] flaps, and 5 flaps were used for upper limb reconstruction [4]. It was not possible to deduce the number of flaps according to the anatomical area in one case [15]. Direct donor site closure was achieved in all the cases [3-17]. Secondary flap debulking was required in 10 papers and was performed through liposuction $[3,7-9,11-13,15,17]$, skin grafting [4], and surgical revision $[6,8,17]$. The authors performing liposuction or revision waited at least 6 months after the primary operation $[3,7-9,11-13,15,17]$, while skin grafting was performed earlier
Fig. 3. The adipofascial variant of the anterolateral thigh flap Adipofascial anterolateral thigh flap, broken down to the level of the vascular pedicle. Note that the vascular branches cross the deep fascia and the skin perpendicularly.

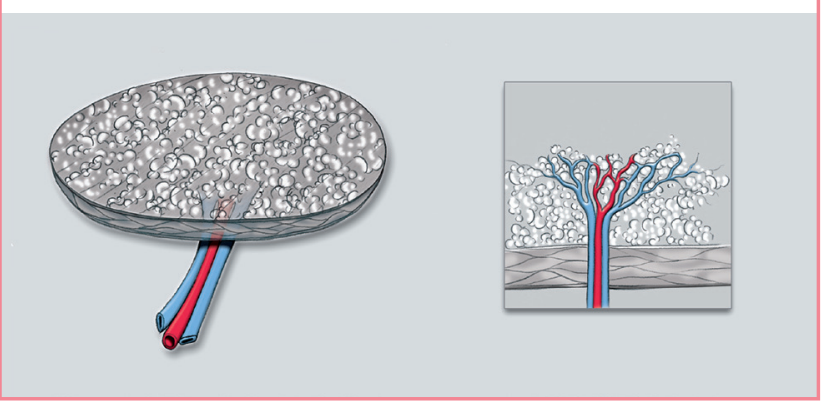

after ALT harvesting [4]. Flap re-suspension was performed in 3 papers, and in all three papers, debulking and suspension were performed simultaneously $[8,12,13]$.

\section{Overall analysis}

Because almost all of the flaps reviewed were transferred to another area of the body using microsurgery, other than Wang et al. who used pedicled flaps [10], we collected a uniform cohort from which to draw conclusions and recommendations. This review demonstrates that secondary debulking and flap sagging are the most frequent complications. Delayed debulking occurred in 32\% (51/163 flaps) of the cases, and flap sagging requiring resuspension occurred in 8\% (13/163 flaps) (Fig. 2). Both complications occurred in patients undergoing reconstructive surgery of the head and neck. Moreover, it appears that adipofascial flaps are primarily limited to volume restoration of the head and neck $[3,6-9,11-15,17]$ but can also be harvested for intraoral $[5,16]$ and limb reconstruction [4-10].

\section{DISCUSSION}

The aim of this study was to systematically identify the common surgical indications, complications and donor site morbidity of the adipofascial variant of the ALT flap (Fig. 3). Primary thinning of the adipofascial ALT flap does not affect the stronger blood supply provided by the subfascial plexus, and this is confirmed by the absence of vascular-related complications. This last issue can be explained because the perforating vessels run perpendicular after passing the deep fascia, as shown in Fig. 1 $[12,18]$. This hair-lacking configuration can be trimmed to 2 $\mathrm{mm}$ and used with sub-optimal results on almost every area of the body [4]. The deep fascia prevents linkage or sagging by anchoring the flap to the underlying structures, such as the periosteum [6]. When ALT flaps are harvested to reconstruct oncologic defects, it is advisable to perform a volume overcorrection 
of approximately $10 \%$ in anticipation of radiation-associated soft tissue atrophy [17]. A small skin island can be harvested for clinical monitoring or to decrease wound tension in the recipient wound bed $[15,17]$. The fat aspirated during secondary debulking can be re-injected to achieve an almost perfect symmetry, which is particularly important for facial correction, or to depress scar revision of the thigh [13]. The absence of different skin textures and colours makes it possible to bury the flap for facial volume correction or to obtain spontaneous mucosalization and to avoid a patch-like appearance $[5,16]$. When used for lower limb reconstruction, ALT flaps provide a gliding surface for tendon movement and also decrease the tendency of the flap to create adhesions [4]. More importantly, superior functional and aesthetic outcomes are achievable with an associated low morbidity at the donor site compared to the adipocutaneous variant of the flap. The ALT flap gained great popularity in mainland China, Taiwan, and Japan based on its versatility, the twoteam approach and low donor-site morbidity. However, due to several variations in the vascular anatomy of the perforators, the ALT flap has not been met with the same enthusiasm in Europe and North America. These disadvantages can be overcome by increased surgical experience and the ability to harvest a thinner flap through accurate suprafascial dissection or thinning the flap in the adipofascial configuration. Donor site morbidity has been reduced through primary closure in almost all of the cases. Although this can create a variable scar depression, it can be easily revised later.

\section{CONCLUSIONS}

The adipofascial ALT flap can be successfully elevated and transplanted for the correction of soft tissue defects of several types and sizes. This flap provides tissue to fill large defects and improve pliability. It has strong and safe blood supply that permits adequate debulking both immediately or delayed without vascular complications. The presence of the deep fascia provides the possibility of preventing sagging by means of suspension and fixation to functional reconstructive purposes as in the intraoral cavity. Donor site morbidity is minimal and can be achieved by immediate direct closure or liposuction and direct closure to mitigate thigh deformity.

\section{REFERENCES}

1. Song YG, Chen GZ, Song YL. The free thigh flap: a new free flap concept based on the septocutaneous artery. $\mathrm{Br} \mathrm{J}$ Plast Surg 1984;37:149-59.

2. Song R, Song Y, Yu Y, et al. The upper arm free flap. Clin
Plast Surg 1982;9:27-35.

3. Guelinckx PJ, Sinsel NK. Facial contour restoration in Barraquer-Simons syndrome using two free anterolateral thigh flaps. Plast Reconstr Surg 2000;105:1730-6.

4. Hsieh CH, Yang CC, Kuo YR, et al. Free anterolateral thigh adipofascial perforator flap. Plast Reconstr Surg 2003;112: 976-82.

5. Agostini V, Dini M, Mori A, et al. Adipofascial anterolateral thigh free flap for tongue repair. Br J Plast Surg 2003;56: 614-8.

6. Masaki F. Correction of hemifacial atrophy using a free flap placed on the periosteum. Plast Reconstr Surg 2003;111: 818-20.

7. Wang X, Qiao Q Liu Z, et al. Free anterolateral thigh adipofascial flap for hemifacial atrophy. Ann Plast Surg 2005;55: 617-22.

8. Wolff KD, Kesting M, Loffelbein D, et al. Perforator-based anterolateral thigh adipofascial or dermal fat flaps for facial contour augmentation. J Reconstr Microsurg 2007;23: 497-503.

9. Tuncali D, Baser NT, Terzioglu A, et al. Romberg's disease associated with Horner's syndrome: contour restoration by a free anterolateral thigh perforator flap and ancillary procedures. Plast Reconstr Surg 2007;120:67e-72e.

10. Wang XC, Lu Q Li XF, et al. Reversed anterolateral thigh adipofascial flap for knee and proximal calf defects. Burns 2008;34:868-72.

11. Agostini T, Agostini V. Adipofascial anterolateral thigh free flap for hemifacial atrophy. Acta Otorhinolaryngol Ital 2009; 29:103-7.

12. Jin $\mathrm{X}$, Teng $\mathrm{L}, \mathrm{Xu}$ J, et al. Anterolateral thigh adipofascial flap for the restoration of facial contour deformities. Microsurgery 2010;30:368-75.

13. Teng L, Jin X, Wu G, et al. Correction of hemifacial atrophy using free anterolateral thigh adipofascial flap.J Plast Reconstr Aesthet Surg 2010;63:1110-6.

14. Zhang Q, Qiao Q, Yang X, et al. Clinical application of the anterolateral thigh flap for soft tissue reconstruction. J Reconstr Microsurg 2010;26:87-94.

15. Zhang Q, Qiao Q, Zhou G, et al. Anterolateral thigh adipofascial flap for correction of facial contour deformities and micromastia. J Reconstr Microsurg 2010;26:341-5.

16. Agostini T, Agostini V, Lazzeri D. Current roles of adipofascial anterolateral thigh flap in head and neck reconstructions. Head Neck 2011;33:595-6.

17. Hanasono MM, Skoracki RJ, Silva AK, et al. Adipofascial perforator flaps for "aesthetic" head and neck reconstruction. Head Neck 2011;33:1513-9. 
18. Agostini T, Agostini V. Adipofascial versus fasciocutaneous anterolateral thigh flap in oral cavity reconstruction: focus on the vascular supply. J Plast Reconstr Aesthet Surg 2009; 62:e633-4. 